\title{
Integrated mechanism reviews
}

doi:10.1038/pr.2012.31

A mong the scientific journals devoted to child health, Pediatric Research focuses on mechanistic aspects of disease etiology, pathogenesis, and interventions (1). In keeping with this mission, we strongly believe that review articles designed to propose, discuss, and/or reject mechanisms are of great interest to a large proportion of our readers. In this editorial, we make the case for what we call integrated mechanism reviews (IMRs), and we invite readers to submit proposals from their field of interest.

\section{Review Articles}

In general, review articles are important cornerstones of biomedical research communication. Readers appreciate them because they provide a comprehensive and, ideally, balanced overview of an important topic. Editors and publishers welcome the added benefit of increased impact factors (2).

Review articles are sometimes considered secondary literature that has made it almost all the way from subjective, unreliable ideas toward objective, reliable knowledge (3). We agree that this might be true for workaday review articles that come close to textbook chapters in tone and content. However, this is not the case for more speculative reviews-for example, opinion pieces and hypothesis papers.

We envision IMRs to be different from general overviews and formal meta-analyses such as Cochrane reviews (4). In essence, IMRs should offer a new way of looking at things by providing the theoretical basis for goal-oriented decisions on how to move ahead in a research field. Their authors should try to help readers understand the collective meaning of previous basic, clinical, and epidemiologic research results, which then guides investigators in designing further studies that test hypotheses. In turn, this will either provide additional support for the proposed mechanistic framework or help to reject it. Thus, an IMR would be interesting and meaningful for those working in pediatric research if it (i) is goal-oriented, (ii) integrates previous results from benchwork, clini- cal research, and epidemiology, (iii) focuses on mechanisms, and (iv) generates at least one novel, testable hypothesis.

\section{Goal Orientation}

Authors of IMRs should have a goal that goes beyond a comprehensive description of what is "known in the field." A good IMR begins with the opposite: a concise description of what is not known and how we might want to think about closing such gap(s). We sometimes tell our students and mentees to grab a bright yellow highlighter and a textbook and mark all sentences that start with "It is still unknown..." or contain similar wording. They should then go into the library and/or online and double-check the desideratum. Is "it" truly unknown? If so, proceed to the next step.

\section{Integrating Basic, Clinical, and Population-Level Findings}

At this point, the creative work begins. It is often best to begin with a figure that can be revised and improved (or discarded!) along the way. Readers interested in causality greatly appreciate graphical methods, which have achieved the reputation of being helpful in model building (5). Particularly when proposed mechanisms are still incomplete, the use of mechanistic figures/schemas (6) can be helpful in identifying and displaying such gaps as targets for future mechanistic research (7). Every IMR should have such a figure as its centerpiece and provide supporting evidence from both basic research and clinical/epidemiologic "people science" in order to cover pathogenetic and etiologic mechanisms, respectively. Only if both perspectives are provided in sufficient detail can the reader appreciate the full scope of the argument and decide whether to pursue the ideas presented.

\section{Focus on Mechanism}

As noted above, we envision IMRs to be especially helpful with respect to biological mechanisms, which have been defined as "entities and activities organized such that they are productive of regular 
changes from start or set-up to finish or termination conditions" (6). Bechtel recently supplemented this view by shifting its focus from sequential organization toward orchestrated, nonsequential functioning of mechanisms because these are better suited to explain living systems (7). In particular, IMRs should attempt to comply with the third sense of "mechanism in biology" in Nicholson's taxonomy, namely, as "a step-by-step explanation of the mode of operation of a causal process that gives rise to a phenomenon of interest" (8).

In basic experimental benchwork, pathophysiologic mechanisms are elucidated by means of intervention, based on manipulationist notions of causal inference (9). In observational epidemiologic studies, associations between exposure characteristics and health outcomes are simply observed and/or put to the test. If such studies are large enough, multivariable modeling can help elucidate mechanisms at the etiologic level. The clinical intervention type of study (randomized clinical trial) is the clinical implementation arm of medical research-again, based on manipulationist logic. In all these scenarios, a wellthought-out IMR can provide solid arguments whether and about how such studies can be performed to come closer to the goal of health improvements. It should go without saying that a special emphasis of IMRs in Pediatric Research should be on developmental mechanisms.

\section{Hypothesis Generation}

The fourth characteristic of an IMR is the generation of at least one testable hypothesis. Such hypotheses outlined in the IMR should be generously supported by data from others, can be bolstered by your own pilot data (but see the "Caveats" section below regarding the angst associated with being scooped), and should be complex enough that readers don't wonder why you haven't tested it yet. The more overarching your theory, the more potential hypotheses it should generate.

\section{Why Theory?}

Some of us are left with the impression that theory does not play as prominent a role in child health research-at least in its biologically oriented disciplines-as in, say, physics. Why do we encourage theoretical work to the degree that we invite submission of proposals for such "thought papers"?

Goodson gives seven reasons for thinking theoretically about health promotion (10). Although all seven are relevant to pediatric research, we believe that two are crucial in our current context. First, she reminds us of the allegory in which pieces of knowledge are individual bricks and theory is the mortar that holds those bricks together (11). Second, she suggests, as do Norgaard et al. (12), that theory can provide road maps for research practice by supplementing descriptive questions (“what?," "how many?”) with "why?" A good IMR would also ask "how?" and propose at least one answer.

\section{Caveats}

Do not write an IMR if you are easily scared of being scooped. Authors of IMRs must be willing to share their ideas even though they might not be the first to receive the grant, publish the paper, earn the fame and glory.

In addition, authors should be ruthlessly subjective. Not only is an objective perspective, currently coming back into fashion (13), difficult to achieve, it is also counterproductive if you want to craft a gripping plaidoyer. Why shouldn't a cogent subjective argument in favor of a proposed mechanism be fruitful? If it is possible to mount a similarly cogent counterargument, the IMR should not be written in the first place.

Authors of IMRs are the advocates of their ideas, not the judges. The latter position is held by the reader, who will not necessarily base his or her decision on one IMR but, ideally, on multiple such pieces written from different perspectives. The ultimate goal is to foster excellent research for the improvement of child health. We eagerly await your proposals.

\section{Olaf Dammann}

Public Health and Community Medicine and Pediatrics, Tufts University School of Medicine, Boston, Massachusetts

\section{Pierre Gressens}

INSERM, U676, Hôpital Robert Debré, Paris, France

\section{REFERENCES}

1. Dammann O, Gressens P. Dear author. Pediatr Res 2011;69:463-4.

2. Garfield E. Which medical journals have the greatest impact? Ann Intern Med 1986;105:313-20.

3. Bauer HH. Scientific literacy and the myth of the scientific method. Urbana, IL: University of Illinois Press, 1992.

4. Higgins JPT, Green S, eds. Cochrane Handbook for Systematic Reviews of Interventions, version 5.1.0. The Cochrane Collaboration, 2011.

5. Greenland S, Brumback B. An overview of relations among causal modelling methods. Int J Epidemiol 2002;31:1030-7.

6. Machamer PK, Darden L, Craver CF. Thinking about mechanisms. Philos Sci 2000;67:1-25.

7. Bechtel W. Mechanism and biological explanation. Philos Sci 2011;78:53357.

8. Nicholson DJ. The concept of mechanism in biology. Stud Hist Philos Biol Biomed Sci, in press.

9. Woodward J. Making things happen: a theory of causal explanation. New York: Oxford University Press, 2003.

10. Goodson P. Theory in health promotion research and practice. Sudbury, MA: Jones \& Bartlett, 2010.

11. Forscher BK. Chaos in the brickyard. Science 1963;142:339.

12. Norgaard LS, Morgall JM, Bissell P. Arguments for theory-based pharmacy practice research. Int J Pharm Pract 2000;8:77-81.

13. Levine S. Rehabilitating objectivity: Rorty, Brandom, and the new pragmatism. Can J Philos 2010;40:567-90. 